\title{
静动态力学条件下多孔金属的材料/结构多级优化 设计研究
}

\author{
刘远东，尹益辉"，郭中泽 \\ 中国工程物理研究院总体工程研究所, 绵阳 621900 \\ *E-mail: yinyh@caep.ac.cn
}

收稿日期: 2011-09-15; 接受日期: 2012-05-07

国家重点基础研究发展计划(“973”计划)(批准号: 2010CB832700)资助项目

\begin{abstract}
摘要将微结构胞元内部构型设计、胞元排布设计和宏、细观跨尺度计算相结合, 建 立了基于同一微结构的材料/结构多目标拓扑优化的静动力学设计模型. 分别给出了材 料设计、结构设计和材料/结构一体化设计的灵敏度有限元列式, 在此基础上以简支梁和 悬臂梁为例进行了数值计算. 结合多孔金属材料具有功能性特点, 基于数值算例的结果, 在结构承载方面讨论了多孔金属材料/结构静动力学优化设计中的材料设计、结构设计和 一体化设计的异同和各自的适用范围. 指出材料设计侧重于功能性, 结构设计侧重于结 构效率, 而材料/结构一体化设计由于兼顾了功能性和结构效率特点, 较好地实现了集成 化设计; 为多孔金属设计选取合适的设计方式提供依据.
\end{abstract}

\author{
关键词 \\ 多孔金属 \\ 多目标 \\ 多级优化 \\ 材料设计 \\ 结构设计 \\ 材料/结构一体化设计
}

传统的泡沫类多孔材料的内部微观结构是无序 的, 泡沫孔的大小也很不规则, 以致泡沫材料的力学 性能差异很大. 随着工程实践的发展, 人们对高性能 材料的需求已经超越了传统的多孔材料. 人们期望 新材料具有优良的结构效率和广泛的多功能应用前 景, 如: 轻质、高强、减振、隔振、降噪、散热以及 多功能化应用等. 以此为背景, 出现了 “有序多孔金 属材料”, 如金属点阵材料和线性多孔材料, 也称”结 构化材料, ${ }^{,[1]}$. 它们可以是开孔或闭孔的, 内部微观 结构是规则可设计的. 由于可设计性, “有序多孔金 属材料”可以同时具备一定的承载能力和减振、隔振、 降噪、散热等多种功能, 正由纯粹的功能材料用途向 结构材料延伸, 最终实现多孔金属的功能性和结构 性集成化. 目前, 针对多孔金属材料兼有功能性和结
构性的材料/结构集成设计还处于起步阶段, 已有的 研究多是对功能性和结构性集成设计工作的简化 ${ }^{[2,3]}$. 鉴于此，本文在有序多孔金属具有功能性的前提下， 在结构承载范畴内, 探讨”有序多孔金属材料”的结 构效率(静、动力学特性)在采用传统设计和一体化设 计的异同.

传统的多孔金属静动力学设计分为材料设计和 结构设计两类, 这里定义为两级设计. 材料设计是假 设在结构宏观尺度上材料微结构均匀分布, 将每个 微结构抽象为胞元, 再对胞元进行设计 ${ }^{[4,5]}$. 由于拓 扑优化设计, 胞元含有孔洞, 而孔洞的存在易于具有 对流、换热、减振、隔振、降噪等功能性特点. 结构 设计是将胞元视为均质材料, 对由均质材料构成的 结构进行拓扑优化设计, 即宏观结构的拓扑优化 ${ }^{[6]}$.

英文版发表信息： Liu Y D, Yin Y H, Guo Z Z. Static and dynamic design based on hierarchical optimization for materials and structure of porous metals. Sci China Tech Sci, 2012, 55: 2808-2814, doi: 10.1007/s11431-012-4917-3 
显然, 材料设计对复杂构型微结构性能的准确表征 具有一定难度, 而结构设计会造成结构的圥余. 针对 传统设计的现状, 人们研究和发展了多孔材料的材 料/结构一体化静力学设计方法 ${ }^{[7 ~ 15]}$, 动力学材料/结 构一体化设计方法还比较少 ${ }^{[16,17]}$, 静动力学材料/结 构一体化设计方法还未见相关文献报道. 多孔金属 静动力学一体化设计是通过控制多孔金属微结构的 内部拓扑与宏观排布来设计该类材料/结构的特定力 学性能. 文献[7 17]对上述三种概念设计方法已有部 分讨论, 但没有系统地讨论其异同和各自的适用范 围. 本文在静动力结构承载范畴内, 探讨“有序多孔 金属材料”各级设计的异同和各自的适用范围.

将微结构胞元设计和多尺度计算 ${ }^{[18]}$ 相结合, 建 立了基于同一微结构的材料/结构一体化静动力学设 计模型, 分别给出了材料设计、结构设计和材料/结构 一体化设计的灵敏度有限元列式. 在此基础上以简 支梁和悬臂梁为例进行了数值计算, 比较了多孔金 属材料/结构静动力学优化设计中的材料设计、结构 设计和材料/结构一体化设计的异同和各自的适用范 围, 为在静动力多目标条件下多孔金属的设计选取 合适的设计方式提供依据.

\section{1 一体化优化设计模型}

\section{1 材料/结构一体化设计数学模型}

首先对微结构胞元尺度和宏观尺度上的设计域 分别进行单元划分, 如图 1 所示. 图 1 中, 定义两组 独立设计变量, 第一组是定义结构划分的超单元的 密度, 称为“宏观密度变量” $P_{i}(i=1, \cdots, M)$, 这里 $P_{i}=1$
表示第 $i$ 个超单元区域存在微结构胞元, $P_{i}=0$ 表示第 $i$ 个超单元区域无微结构胞元. 第二组是定义每个超 单元内划分的有限元网格密度, 称为“微观密度变 量” $\rho_{j}(j=1, \cdots, n)$, 类似地, $\rho_{j}=1$ 表示第 $j$ 个有限单元 区域存在实体材料, $\rho_{j}=0$ 表示第 $j$ 个有限单元区域 无材料. 同时定义每个超单元中对应位置的有限单 元的微观密度变量值相同. 根据这样的网格划分和 变量定义, 最终第 $i$ 个超单元内第 $j$ 个有限单元所在 区域的材料密度 $x_{i j}{ }^{[19]}$

$$
x_{i j}=P_{i} \rho_{j} .
$$

采用常见的 RAMP(Rational Approximation of Material Properties)模型, 有限单元材料的弹性模量 和质量的插值格式为

$$
\begin{gathered}
E=\frac{x}{1+\alpha(1-x)} E_{0}, \\
M=x M_{0},
\end{gathered}
$$

其中 $E_{0}$ 和 $M_{0}$ 分别为实体材料的弹性模量和质量, $x \in[0,1]$ 为单元材料的伪密度值, $\alpha$ 为惩罚因子, 对 介于 0 和 1 之间的伪密度值进行惩罚, 参照文献[20], 取其值为 $\alpha=3$. 对结构设计区域进行有限单元离散 后，采用敏度过滤技术抑制有限元计算中的棋盘格 现象 ${ }^{[20]}$.

为了处理不同性质的结构响应量在数量级上的 差异, 需要将各目标函数进行归一化. 具体就是: 设 单目标优化后, 初始结构柔度最小值为 $\bar{C}$, 基频特征 值最大为 $\overline{\lambda_{k}}$, 则以静态柔顺度最小和动态特征值最 大为综合目标 $D$ 的优化模型为

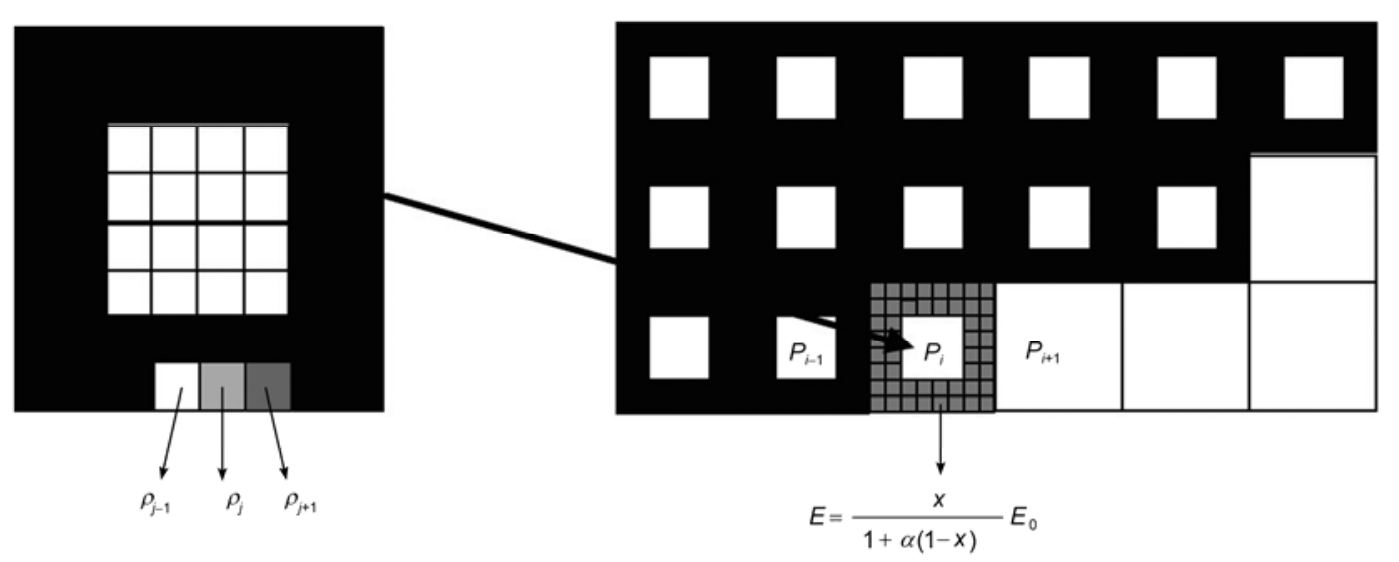

图 1 微结构胞元微观构型和宏观排布的材料/结构一体化优化设计示意图 
Find $P_{i}(x)(i=1,2, \cdots, M), \rho_{j}(x)$

$(j=1,2, \cdots, n)$,

Min $D=\beta \frac{\overline{\lambda_{k}}}{\lambda_{k}(x)}+(1-\beta) \frac{C(x)}{\bar{C}}$,

$\left(k=1,2, \cdots, n_{\text {dof }}\right)$,

Constraint I : $\left(K-\lambda_{k} M_{1}\right) \phi_{k}=0$,

Constraint II : $\sum_{i=1}^{M} \sum_{j=1}^{n} P_{i} \rho_{j} v_{j}-V_{0}=0$,

Constraint III $: \sum_{j=1}^{n} \rho_{j} v_{j}-V_{1}=0$,

Constraint IV: $\quad 0<P_{\min } \leqslant P_{i} \leqslant P_{\text {max }}, i=1, \cdots, M$, $0<\rho_{\min } \leqslant \rho_{j} \leqslant \rho_{\max }, j=1, \cdots, n$,

其中 $\beta$ 是权重系数, $C$ 表示结构的柔顺度; 约束 I 是 自由振动控制方程, $\lambda_{k}$ 是结构 $k$ 阶圆频率的平方(特 征方程的特征值), $\phi_{k}$ 是结构的第 $k$ 阶特征向量, 两 者形成特征对, $M_{1}$ 为结构的质量矩阵; 约束 II 给出 了实体材料用量上限, $v_{j}$ 为超单元中第 $j$ 单元的体积, $V_{0}$ 为给定的实体材料用量; 约束III给出了胞元用量 上限, $V_{1}$ 为超单元的材料用量; 约束 $\mathrm{IV}$ 是两类密度变 量的上、下限约束, $P_{\text {min }}$ 和 $\rho_{\text {min }}$ 为趋于 0 的正数 (取 $\left.10^{-3}\right)$, 以避免计算中出现刚度矩阵奇异, $P_{\text {max }}$ 和 $\rho_{\text {max }}$ 分别是超单元和有限单元的最大密度值.

文中提出的综合目标优化模型是通过对单个目 标归一化, 然后线性加权得到的. 该模型可有效避免 出现一个目标函数支配另一个目标函数的情况，通 过敏度过滤技术在一定程度上抑制复杂拓扑结构的 出现，但该方法主要适用于凸问题的求解，本文的问 题亦是凸问题. 同时, 我们会发现若在(4)式中不加 约束条件III, 则在结构范畴内, 最优的微结构形式往 往是各向同性的实心材料 ${ }^{[2]}$, 这是因为本文的设计方 法是建立在微结构均匀同一的基础上的. 而孔洞的 存在易于使结构具有对流、换热、减振、隔振、降噪 等功能性特点, 因此在要求是多孔材料设计的前提 条件下, 模型中添加了约束条件III.

\section{2 各级优化设计的灵敏度列式}

材料/结构一体化设计的灵敏度即是目标函数对 各设计变量的导数, 其列式为

$$
\begin{aligned}
\frac{\partial D}{\partial P_{i}}= & \frac{\beta \overline{\lambda_{k}}}{P_{i}\left(\lambda_{k}(x)\right)^{2}} \sum_{j=1}^{n}\left[\operatorname{Dsene}_{i j} \frac{1+\alpha}{\left[1+\alpha\left(1-x_{i j}\right)\right]}-\text { Dkene }_{i j}\right] \\
& -\frac{(1-\beta)(1+\alpha)}{\left[1+\alpha\left(1-x_{i j}\right) P_{i} \bar{C}\right.} \sum_{j=1}^{n} \text { Sene }_{i j}, \\
\frac{\partial D}{\partial \rho_{j}}= & \frac{\beta \overline{\lambda_{k}}}{\rho_{j}\left(\lambda_{k}(x)\right)^{2}} \sum_{i=1}^{M}\left[\operatorname{Dsene}_{i j} \frac{1+\alpha}{\left[1+\alpha\left(1-x_{i j}\right)\right]}-\text { Dkene }_{i j}\right] \\
& -\frac{(1-\beta)(1+\alpha)}{\rho_{j} \bar{C}\left[1+\alpha\left(1-x_{i j}\right)\right]} \sum_{i=1}^{M} \text { Sene }_{i j},
\end{aligned}
$$

其中 Sene $_{i j}$ 为第 $i$ 个超单元中第 $j$ 个有限单元的应变能, Dsene $_{i j}$ 和 Dkene $e_{i j}$ 分别为第 $i$ 个超单元中第 $j$ 个有限单 元的应变能和动能. 柔顺度和特征值对两尺度密度 的灵敏度建立在后处理得到的单元应变能和动能的 基础上, 因此有相对高的计算效率. 同时, $P_{i}=1$ 表 示一体化设计退化到微结构设计, 但更好的保留了 载荷和约束边界条件; $\rho_{j}=1$ 即为微结构满铺, 相当 于宏观结构优化.

在(5)和(6)式中, 若取 $\rho_{j} \equiv 1$, 就得到由材料/结 构一体化设计退化而来的单纯结构设计的灵敏度列 式:

$$
\begin{aligned}
\frac{\partial D}{\partial \rho_{j}}= & \frac{\beta \overline{\lambda_{k}}}{\rho_{j}\left(\lambda_{k}(x)\right)^{2}} \sum_{i=1}^{M}\left[\text { Dsene }_{i j} \frac{1+\alpha}{\left[1+\alpha\left(1-x_{i j}\right)\right]}-\text { Dkene }_{i j}\right] \\
& -\frac{(1-\beta)(1+\alpha)}{\rho_{j} \bar{C}\left[1+\alpha\left(1-x_{i j}\right)\right]} \sum_{i=1}^{M} \text { Sene }_{i j} .
\end{aligned}
$$

在(5)和(6)式中, 若取 $P_{i} \equiv 1$, 就得到由材料/结 构一体化设计退化而来的单纯材料设计的灵敏度列 式:

$$
\begin{aligned}
\frac{\partial D}{\partial P_{i}}= & \frac{\beta \overline{\lambda_{k}}}{P_{i}\left(\lambda_{k}(x)\right)^{2}} \sum_{j=1}^{n}\left[\operatorname{Dsene}_{i j} \frac{1+\alpha}{\left[1+\alpha\left(1-x_{i j}\right)\right]}-\text { Dkene }_{i j}\right] \\
& -\frac{(1-\beta)(1+\alpha)}{\left[1+\alpha\left(1-x_{i j}\right)\right] P_{i} \bar{C}} \sum_{j=1}^{n} \text { Sene }_{i j} .
\end{aligned}
$$

\section{3 设计变量的迭代格式}

基于变分原理的泛函形式, 引入 Lagrange 乘子 构造 Lagrange 函数, 将有约束的问题转化为无约束 的极值问题, 求解目标函数满足一系列约束条件的驻 值问题,基于 Karush-Kuhn-Tucker (KKT) 条件, 结合 文献[21], 可得如下变量更新的迭代格式: 


$$
x_{e}^{k+1}=
$$

$$
\left\{\begin{array}{l}
\max \left((1-m) x_{e}^{k}, x_{\min }\right), \\
\quad \text { if } x_{e}^{k}\left(B_{e}^{k}\right)^{\xi} \leqslant \max \left((1-m) x_{e}^{k}, x_{\min }\right), \\
x_{e}^{k}\left(B_{e}^{k}\right)^{\xi}, \\
\text { if } \max \left((1-m) x_{e}^{k}, x_{\min }\right) \leqslant x_{e}^{k}\left(B_{e}^{k}\right)^{\xi} \leqslant \min \left((1+m) x_{e}^{k}, 1\right), \\
\min \left((1+m) x_{e}^{k}, 1\right), \quad \text { if } \quad x_{e}^{k}\left(B_{e}^{k}\right)^{\xi} \geqslant \min \left((1+m) x_{e}^{k}, 1\right),
\end{array}\right.
$$

其中 $\xi$ 为阻尼系数, 文中取 $0.5 ; m$ 为移动极限的量, 可以在 $0 \sim 1$ 之间变化, 本文取 $0.3, x_{e}$ 为单元 $e$ 的伪密 度变量; 而

$$
B_{e}^{k}=\max \left(0,-\frac{\partial[C(\boldsymbol{X})]}{\partial x_{e}} / \sum_{i=1}^{M} \lambda_{i} \frac{\partial[V(\boldsymbol{X})]}{\partial x_{e}}\right),
$$

其中 $\lambda_{i}$ 为约束函数的拉氏算子.

\section{2 数值算例和结果分析}

\subsection{MBB 梁}

设计区域如图 2 所示, 为一简支梁, 其长 $L=640$, 宽 $H=160$, 厚 $t=5$, 梁的顶端面 $1 / 2$ 处受集中力 $F=-200$ 的作用, 梁的下端面 $1 / 4,3 / 4$ 处分别受集中质 量 $m=2$ 的作用. 基体材料的弹性模量 $E=4 \times 10^{4}$, $\rho=7.8 \times 10^{-6}$, 泊松比 $v=0.3$. 这里各物理量均为无 量纲量. 采用平面 4 节点单元划分网格, 宏观设计区 域的网格为

$16 \times 8$, 微结构的网格为 $20 \times 20, \beta_{1}$ 和 $\beta_{2}$ 分别表 示动、静态目标的权重系数. 结构设计和材料设计中, 材料宏观用量上限为 $\zeta_{1}=0.4$, 材料/结构一体化多目 标设计中, 材料的宏观用量上限为 $\zeta_{1}=0.4$, 微观用量 上限为 $\zeta_{2}=0.8$. 表 1 给出了不同静动态目标的权系数 条件下, 不同级优化设计得到的结构构型和优化目 标结果. 其中, $f$ 表示结构基频, $C$ 表示结构柔顺度.

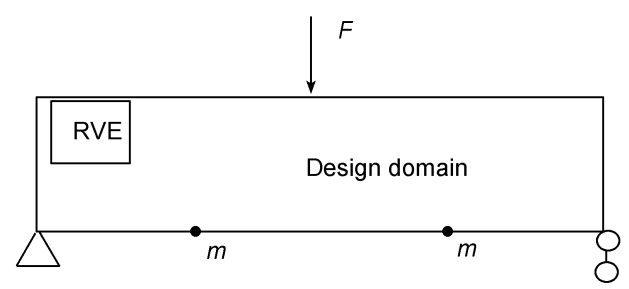

图 2 MBB 梁的结构设计区域和微结构胞元
由于是对称结构, 表中给出了一半结构的形式.

三种权系数下, MBB 梁一体化设计结果(柔顺度 和基频)明显优于基于单一材料微结构的材料设计的 结果, 劣于结构设计的宏观优化设计结果. 并且对应 同类型设计的情况, MBB 梁结构的静动态目标函数 的性能随着相应的权系数的增加, 变得更好, 随着相 应权系数的减少, 变得较差. 在材料设计和材料/结 构一体化设计中, 微结构的构型具有明显的不同; 结 构设计中, 微结构的构型为实心材料.

\section{2 悬臂梁}

设计区域为左端固支的悬臂梁，如图 3 所示，其 长 $L=320$, 宽 $H=180$, 厚 $t=5$. 梁在右边中点受集中质 量 $m=2$ 的作用, 右下角顶点受集中力 $F=-100$ 的作用. 基体材料参数、权重系数和单元种类与上文中的相同, 宏观设计区域的网格为 $16 \times 9$, 微结构的网格为 $20 \times 20$ 结构设计和材料设计中，材料宏观用量上限为 $\zeta_{1}=0.5$, 材料/结构一体化多目标设计中, 材料的宏 观用量上限为 $\zeta_{1}=0.5$, 微观用量上限为 $\zeta_{2}=0.8$. 表 2 给出了不同静动态权系数和不同级下优化的结构构 型和目标函数.

三种权系数下, 悬臂梁一体化设计结果(柔顺度 和基频)明显优于基于单一材料微结构的材料设计的 结果, 劣于结构设计的宏观优化设计结果. 并且对应 同类型设计的情况, 悬臂梁结构的静动态目标函数 的性能随着相应的权系数的增加, 变得更好, 随着相 应权系数的减少, 变得较差. 在材料设计和材料/结 构一体化设计中, 微结构的构型具有明显的不同; 结 构设计中, 微结构的构型为实心材料.

\section{3 讨论}

多孔金属具有功能性特点, 并正从功能性向兼 有功能性和结构性过渡. 然而, 兼有功能性和结构性 的材料/结构集成设计还比较困难. 基于多孔材料具 有功能性的特点, 本文在结构承载范畴内开展多孔 金属静动力学特性的多级优化设计研究, 旨在多目 标、两尺度条件下，为多孔金属提供最优的宏观结构 和微观拓扑. 由上面给出的两个多孔金属柔顺度和 频率设计的各级优化算例可以看出：在结构效率范 畴下，一体化设计的结果明显优于材料设计，略低于 
表 1 MBB 梁不同权系数和不同级下的优化结果

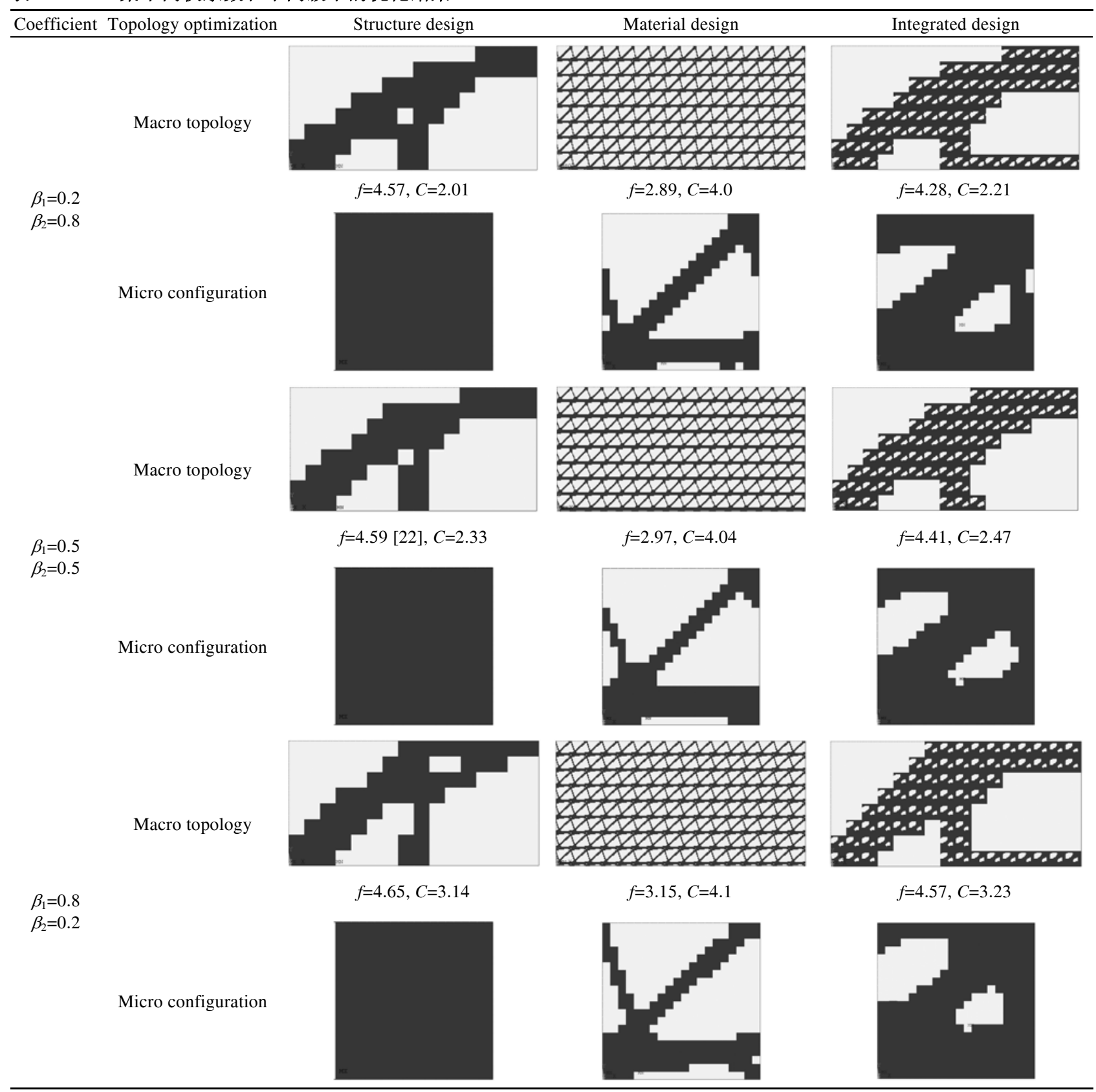

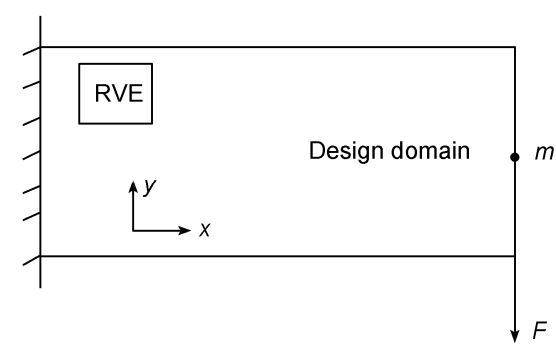

图 3 悬臂梁结构设计区域和微结构胞元
结构设计, 但由于具有孔洞, 更易于具有了功能性的 特点, 即注意了材料与结构的匹配性; 结构设计的结 构效率最高, 但其为实心材料, 不易于具有功能性; 材料设计则主要保留其功能性.

\section{4 结论}

1) 在探讨了结构和材料两个尺度协同优化算 
表 2 悬臂梁不同权系数和不同级下的优化结果

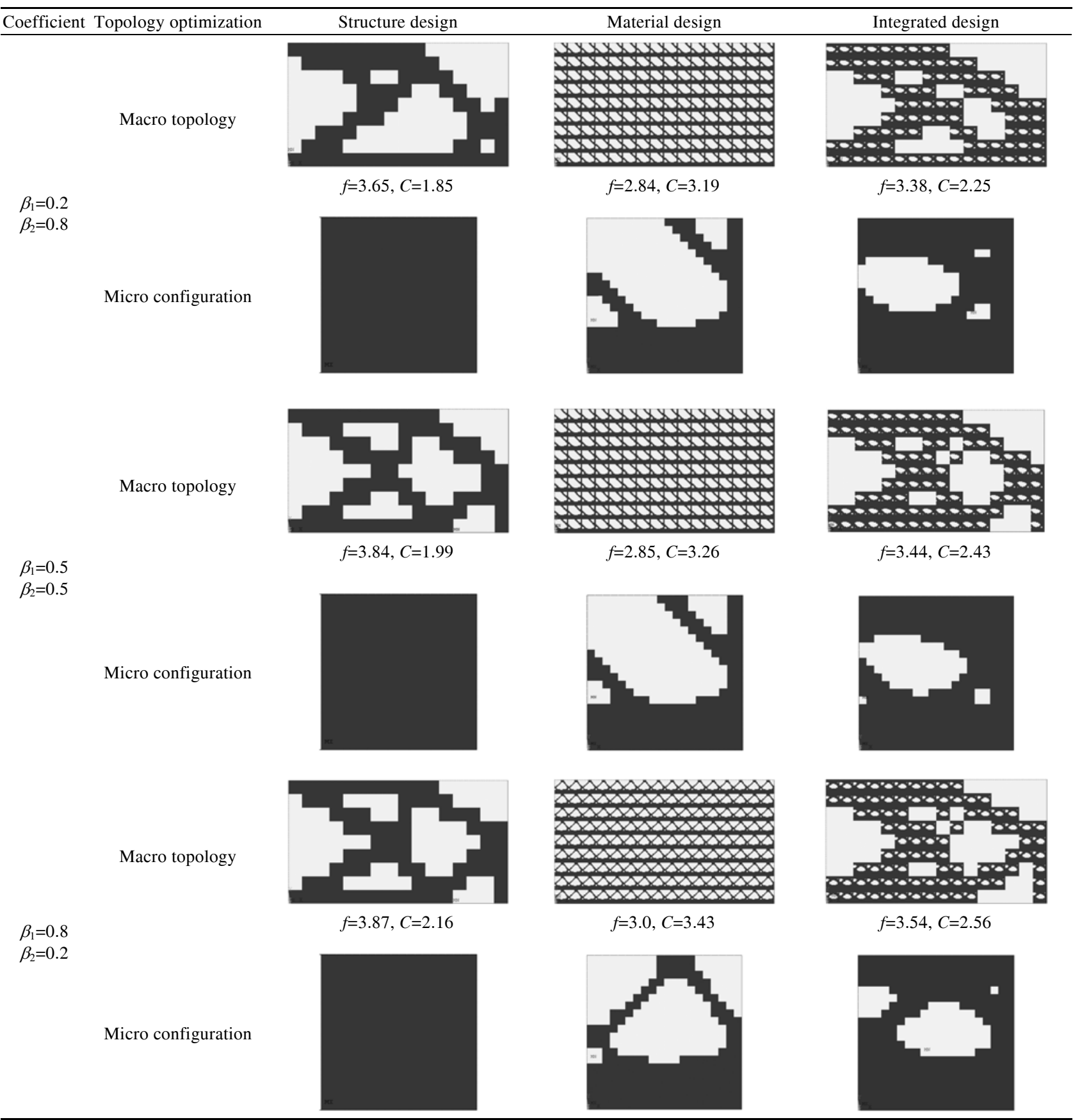

法的基础上, 建立了考虑静动力学特性的材料/结构 一体化设计模型, 并在此基础上, 开展了材料/结构 静动力学条件下的多级优化设计, 给出了静动力学 多目标、多级条件下多孔金属的最优宏观结构和微观 拓扑.

2) 通过算例, 讨论了结构承载范畴下, 各级优
化设计的异同和适用范围, 并得到一体化设计侧重 于材料与结构的匹配性，集中体现出集成设计，即同 时具有功能性, 结构效率损失的又小; 结构设计的结 构效率最高, 为实心材料, 不易于具有功能性; 材料 设计则主要关注其功能性. 这些为针对不同工程目 的的多孔金属设计选取合适的设计方式提供了依据. 


\section{参考文献}

1 卢天健，何德坪，陈常青，等. 超轻多孔金属材料的多功能特性及应用. 力学进展, 2006, 36(4): 517-535

2 阎军, 邓佳东, 程耿东. 基于柔顺性与热变形双目标的多孔材料与结构几何多尺度优化设计. 固体力学学报, 2011, 32(2): 119-132

3 刘涛, 邓子辰. 考虑材料设计变量的热-固耦合结构的优化设计. 固体力学学报, 2006, 27(4): 374-378

4 Simone A E, Gibson L J. Effects of solid distribution on the stiffness and strength of metallic foams. Acta Mater, 1998, 46: 2139-2150

5 Wang A J, McDowell D L. In-plane stiffness and yield strength of periodic metal honeycombs. J Eng Mater Technol, 2004. 137-156

6 刘辛军，李枝东，陈祥. 多工况拓扑优化问题的一种新解法. 中国科学：技术科学, 2011, 41: 920-928

7 张卫红, 孙士平. 多孔材料/结构尺度关联的一体化拓扑优化技术. 力学学报, 2006, 38(4): 522-529

8 Pedersen P. On optimal shapes in materials and structure. Struct Multidiscip O, 2000, 19: 169-182

9 Rodrigues H, Guedes J M, Bendsoe M P. Hierarchical optimization of material and structure. Struct Multidiscip O, 2002, 24: 1-10

10 Evans A G, Hutchinson J W, Ashby M F. Multi-functionality of cellular metal systems. Prog Mater Sci,1999, 43: 171-221

11 Evans A G, Hutchinson J W, Fleck N A, et al. The topological design of multifunctional cellular metal. Prog Mater Sci, 2001, 46: 309-320

12 Thomas J P, Qidwai M A. Mechanical design and performance of composite multifunctional materials. Acta Mater, 2004, 52(8): 2155-2164

13 Soto C A. Simultaneous design of structural topology and material properties. AIAA J, 2000, 4913: 1-9

14 Xia Q, Wang M Y. Simultaneous optimization of material property and topology of functionally graded structures. Comput Aided Design, 2008, 40(6): 660-675

15 Fujii D, Chen B C, Kikuchi N. Composites material design of two-dimensional structures using the homogenization method. Int J Num Meth Eng, 2001, 50: 2031-2051

16 刘远东, 尹益辉, 郭中泽. 尺度关联的微结构构型与排布的材料/结构动力学设计. 复合材料学报, 2011, 28(4): 180-184

17 Niu B, Yan J, Cheng G D. Optimum structure with homogeneous optimum cellular material for maximum fundamental frequency. Struct Multidiscip O, 2009, 29(2): 115-132

18 Bensoussan A, Lions J L, Papanicolaou G. Asymptotic Analysis for Periodic Structures. Amsterdam: North Holland, 1978

19 阎军, 刘岭, 刘晓峰, 等. 考虑尺寸效应的模块化结构两层级优化设计. 力学学报, 2010, 42(2): 268-274

20 Sigmund O. Morphology-based black and white filters for topology optimization. Struct Multidiscip O, 2007, 33: 401-424

21 Bendsoe M P. Optimization of Structural Topology, Shape and Material. Berlin: Springer, 2003

22 龙凯, 左正兴, 间清东. 静动态多目标下的连续体结构拓扑优化. 宇航学报, 2008, 29(2): 456-460 